\title{
Commissioning and first light results of an L'-band vortex coronagraph with the Keck II adaptive optics NIRC2 science instrument
}

\author{
Bruno Femenía Castelláa ${ }^{\mathrm{a}}$, Eugene Serabyn ${ }^{\mathrm{b}}$, Dimitri Mawet ${ }^{\mathrm{c}}$, Olivier Absil ${ }^{\mathrm{d}}$, Peter \\ Wizinowich $^{\mathrm{a}}$, Keith Matthews ${ }^{\mathrm{c}}$, Elsa Huby ${ }^{\mathrm{d}}$, Michael Bottom ${ }^{\mathrm{c}}$, Randy Campbella ${ }^{\mathrm{a}}$, Dwight \\ Chan $^{\mathrm{a}}$, Brunella Carlomagno ${ }^{\mathrm{d}}$, Sylvian Cetre $^{\mathrm{a}}$, Denis Defrère ${ }^{\mathrm{e}}$, Christian Delacroix ${ }^{\mathrm{f}}$, Carlos \\ Gomez Gonzalez ${ }^{\mathrm{d}}$, Aïssa Jolivet ${ }^{\mathrm{d}}$, Mikael Karlsson ${ }^{\mathrm{g}}$, Kyle Lanclos ${ }^{\mathrm{a}}$, Scott Lilley ${ }^{\mathrm{a}}$, Steven \\ Milner $^{\mathrm{a}}$, Henry $\mathrm{Ngo}^{\mathrm{c}}$, Maddalena Reggiani ${ }^{\mathrm{d}}$, Julia Simmons ${ }^{\mathrm{a}}$, Hien Tran ${ }^{\mathrm{a}}$, Ernesto Vargas \\ Catalan $^{\mathrm{g}}$, and Olivier Wertz ${ }^{\mathrm{d}}$ \\ ${ }^{a}$ W. M. Keck Observatory, 65-1120 Mamalahoa Hwy., Kamuela, HI 96743, USA \\ b Jet Propulsion Laboratory, 4800 Oak Grove Dr., Pasadena, CA 91109, USA \\ ${ }^{\mathrm{c}}$ California Institute of Technology, 1200 E. California Blvd, Pasadena, CA 91125, USA \\ ${ }^{\mathrm{d}}$ Department of Astrophysics, Geophysics and Oceanography, University of Liège, 17 allé du \\ Six Août, B-4000 Sart-Tilman,Belgium \\ eSteward Observatory, University of Arizona, 933 N. Cherry Avenue, 85721 Tucson, USA \\ ${ }^{f}$ Sibley School of Mechanical and Aerospace Engineering, Cornell University, Ithaca, USA \\ gAngströmlaboratoriet, Lägerhyddsvägen 175237 Uppsala, Swedem
}

\begin{abstract}
On March 2015 an L'-band vortex coronagraph based on an Annular Groove Phase Mask made up of a diamond sub-wavelength grating was installed on NIRC2 as a demonstration project. This vortex coronagraph operates in the L' band not only in order to take advantage from the favorable star/planet contrast ratio when observing beyond the $\mathrm{K}$ band, but also to exploit the fact that the Keck II Adaptive Optics (AO) system delivers nearly extreme adaptive optics image quality (Strehl ratios values near 90\%) at $3.7 \mu \mathrm{m}$. We describe the hardware installation of the vortex phase mask during a routine NIRC2 service mission. The success of the project depends on extensive software development which has allowed the achievement of exquisite real-time pointing control as well as further contrast improvements by using speckle nulling to mitigate the effect of static speckles. First light of the new coronagraphic mode was on June 2015 with already very good initial results. Subsequent commissioning nights were interlaced with science nights by members of the VORTEX team with their respective scientific programs. The new capability and excellent results so far have motivated the VORTEX team and the Keck Science Steering Committee (KSSC) to offer the new mode in shared risk mode for 2016B.
\end{abstract}

Keywords: W.M. Keck Observatory, NIRC2, high-contrast imaging, coronagraph, vortex phase mask

\section{INTRODUCTION}

At the time of writing this paper, more than 3400 exoplanets have been discovered*. It is generally accepted that direct imaging of planets provides the most unambiguous detection, while eventually providing key information on the planets which is model free. Yet, the largest fraction of exoplanet detections has been achieved by indirect methods, mostly via transiting methods or radial velocities techniques, with a very tiny fraction (less than $2 \%$ ) of exoplanets having been actually imaged. The reason for this lies in the extraordinary technological challenges associated with the large flux ratio between the host star and its planets as well as the small angular separation between star and planets. Due to these difficulties, the vast majority of exoplanets imaged so far are young large

Send e-mail correspondence to B.F.C. at bfemenia@keck.hawaii.edu

*The Extrasolar Planets Encyclopedia, accessed on May 24th 2016, http://exoplanet.eu/ 
Jovian exoplanets (self luminous planets with a much larger thermal emission than what their reflect from their parent stars) around nearby host stars ( 88\% imaged exoplanets are within less than 150 pc from the Sun) making possible to be resolved by current 8-10 $\mathrm{m}$ class telescopes.

Detecting planets this way requires high contrast imaging systems coupled to adaptive optics systems. Nearly all high contrast imaging systems rely on the use of a coronagraphic system, and among all the families of coronagraphs the vortex coronagraphs ${ }^{1}$ have been particularly promising due to their excellent properties ${ }^{2}$ in terms of very small Inner Working Angle (IWA), high transmission, clear $360^{\circ}$ discovery space, low chromatic dependence and ease of implementation in existing coronagraphic systems.

After successful lab tests ${ }^{3}$ of the AGPM L-band vortices, with contrast ratios of $6 \times 10^{-5}$ achieved at $2 \lambda / D$, the technology is being adopted by large 8-m diameter monolithic primary mirror class telescopes: VLT, ${ }^{4} \mathrm{LBT}^{5}$ and Subaru. ${ }^{6}$ Despite the first on-sky tests occurring with small telescopes and subapertures in the visible ${ }^{7}$ and near-infrared8 the current generation of AGPM-based vortex coronagraphs has been optimized to work in the thermal IR L' and M bands. The reasons for this choice are multiple: (1) the trade-off between the decrease in sensitivity caused by the IR sky background but the most favorable contrast ration star-planet in the IR (2) the increase of the vortex performance due to the excellent image quality achieved in L' and M. Notice that Adaptive Optics (AO) systems designed mainly for observations in the Near-InfraRed (NIR) when feeding systems in L' provide AO corrections in the 80-90\% Strehl Ratio (SR) domain that in the NIR bands require Extreme Adaptive Optics (ExAO, e.g. PALM-300 in the Hale telescope, ${ }^{9}$ GPI in Gemini, ${ }^{10}$ SPHERE ${ }^{11}$ in VLT or SCExAO in Subaru ${ }^{6}$ ).

Here we present the details of the commissioning of an Annular Groove Phase Mask (AGPM) vector vortex coronagraph with the Adaptive Optics (AO) fed NIRC2 instrument at the segmented 10-m diameter Keck II telescope. In this paper we report the steps taken to commission this new NIRC2 observing mode in terms of hardware, calibration and operational software developments. We also provide highlights of preliminary scientific results achieved during the vortex commissioning.

\section{THE VORTEX AT NIRC2 ON KECK II TELESCOPE}

With the new generation of ExAO systems ${ }^{6,10,11}$ coming online, consolidated first generation AO systems still have an important role in imaging exoplanets. First generation AO instruments designed and operated in the NIR to routinely achieve $\mathrm{SR} \gtrsim 50 \%$ on bright targets under good seeing conditions become ExAO systems when operated in L', compensate the loss of sensitivity due to larger backgrounds in the thermal IR bands with a better contrast ratio star/planet and, in the case of the vortex coronagraph, the small IWA values $(\sim 0.9 \lambda / D)$ partially compensate the loss of angular resolution when going to longer wavelengths.

An important example of this are the Keck telescopes: despite that the Keck II AO instrument NIRC2 has played a key role in the emerging field of exoplanet direct imaging (e.g. see Refs. 12,13) and being the largest telescopes equipped with $\mathrm{AO}$, no current plans exist to upgrade the Keck AO systems with an ExAO facility. In this context the installation of a vortex in NIRC2 may alleviate the situation by improving the Keck exoplanet imaging capabilities. NIRC2 is a near-infrared imager behind the Keck II AO system, providing three selectable cameras with pixel scales of 10,20 and 40 mas/pixel to cover the wavelength range 1 to $5 \mu m$. Two filter wheels with 18 positions each provide a variety of narrow and broad-band filters and/or grisms, while a focal plane mechanism provides slits and occulting spots for coronagraphy. A dedicated slide carries larger grisms for spectroscopy. Six selectable pupil masks are available to reduce background noise sources; four of these rotate in concert with the telescope pupil and one is specific to spectroscopy. The detector is a $1024 \times 1024$ Aladdin-3 $\mathrm{InSb}$ array.

\subsection{Installation of an internal IR calibration source}

NIRC2 was designed and deployed with NIR and thermal IR imaging capabilities up to L and M bands ${ }^{\dagger}$. However, it was not until we initiated this project that the Keck II AO system could have access to an internal IR source.

\footnotetext{
${ }^{\dagger}$ IR wide filters in NIRC2 operate at L-wide with $\lambda=3.5197 \pm 1.3216 \mu \mathrm{m}, \mathrm{L}$ ' with $\lambda=3.776 \pm 0.700 \mu \mathrm{m}$ and M-short with $\lambda=4.670 \pm 0.241 \mu \mathrm{m}$, and narrow band filters Brackett gamma continuum with $\lambda=3.987 \pm 0.069 \mu \mathrm{m}$ and Brackett alpha at $\lambda=4.052 \pm 0.068 \mu \mathrm{m}$
} 

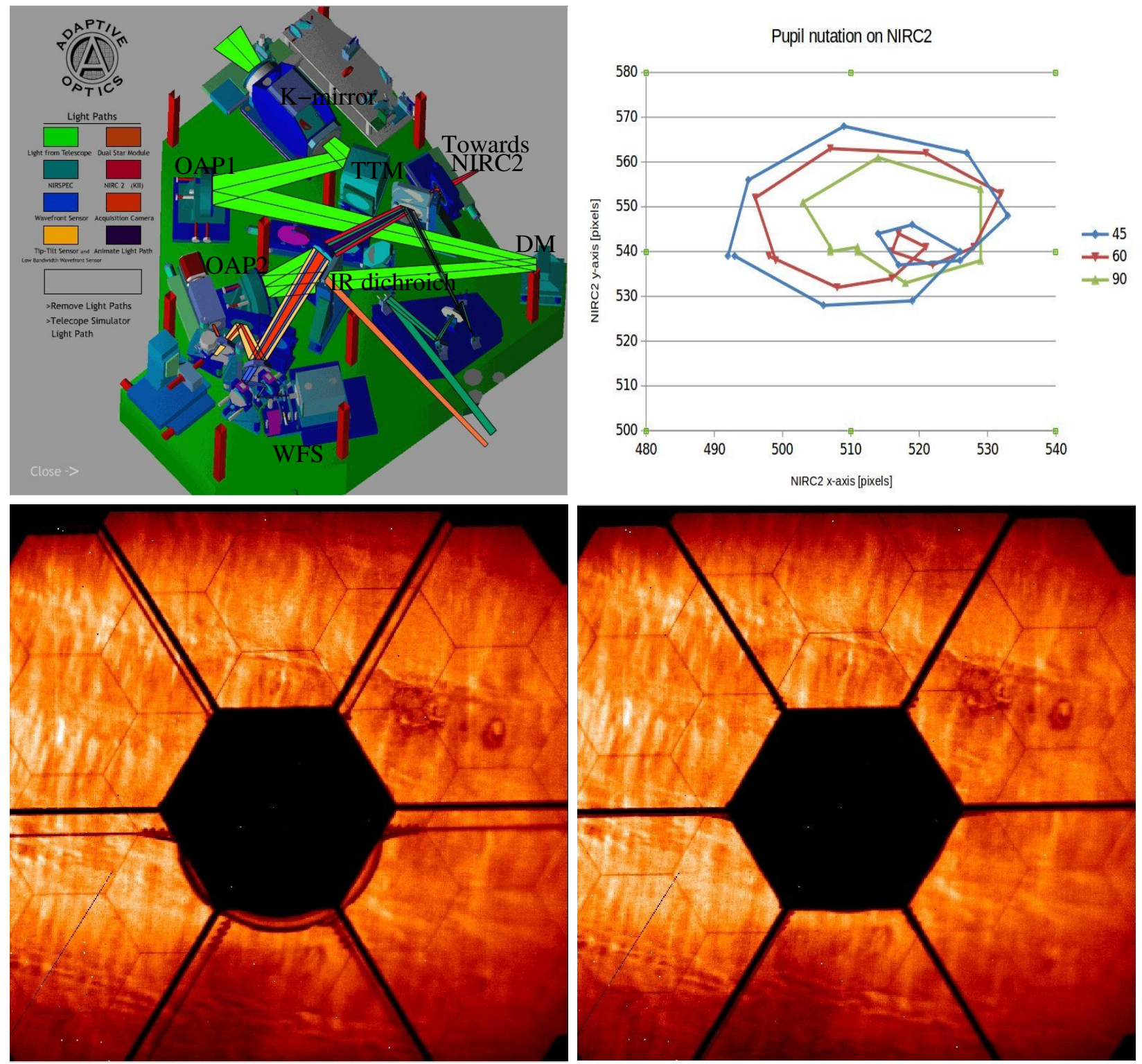

Figure 1. Top Left: 3D view of the Keck II AO bench with labels on the elements requiring re-alignment (K-mirror, OAPs \& IR dichroic) as well as the Tip-Tilt Mirror (TTM), the Deformable Mirror (DM) and the WaveFront Sensor (WFS); NIRC2 is located on the right. Top Right: pupil nutation as measured before re-alignment of first mirror in the K-mirror assembly; different colors correspond to the tube telescope at 45, 60 and $90^{\circ}$ elevation. Bottom Left: Example of telescope pupil and NIRC2 pupil mask misalignment; here using the largehex pupil which is nearly identical to the actual M1 shape on the outer edge but the inner obscuration is not a circle but an hexagon. Bottom Right: Example of good alignment $(\sim 1 \%)$ after adjustments on the Keck II AO bench.

Standard AO calibrations and the Non-Common Path Aberration calibrations (NCPA, this comprising image sharpening and WFS calibration) are performed with a white light source providing no light at L and M bands. Since our vortex Focal Plane Mask (FPM) is designed and optimized for operation in L \& M, it was mandatory to have access to an IR source. The solution implemented relied on off-the-shelf parts ${ }^{\ddagger}$ and included a broad-

${ }^{\ddagger}$ From Thor Labs, part numbers: SLS202 for the IR source, P1-23Z-FC-2 and P1-23Z-FC-5 for the IR fibers, FW102C for the filter wheel, NENIRXX ND filters (XX=01, 02, 03, 04, 05, 06, 10, 13, 20, 30, 40, 50 60) and SLSC2 for the collimators 
band Tungsten source, single mode IR fibers (low wavelength cut-off at $\sim 2.3 \mu \mathrm{m}$ ), a six position filter wheel accommodating 4 IR ND=1.3, 3.0, 4.0 \& 5.0 filters (plus an open and a blocked position), and two collimators in the assembly with the Tungsten source and filter wheel. The initial purpose of this IR source was to validate the image quality of NIRC2 at L \& M bands, and eventually upon deploying the speckle nulling code it was a needed part for the calibration procedure (see Section 2.5) .

\subsection{Preparing the Keck II AO bench}

A series of steps were required on the Keck II AO bench in order to optimize the image quality and pupil registration:

Re-alignment of optical derotator It had been noticed for some time the NIRC2 pupil masks were not aligned with the Keck 2 secondary mirror and spiders. After the K-mirror was coated, re-installed and presumably re-aligned a significant pupil nutation was observed, which was determined to be caused by the 1st mirror on the K-mirror assembly. Re-adjustment of the 1st mirror took place during September 2014 and the pupil nutation was reduced to $\sim 1 \%$ of the pupil. Next, the off-axis parabolas (OAPs) were synchronously adjusted in order to center the secondary on the pupil mask.

Re-orientation of the IR dichroic Chromatic elongation on NIRC2 had been an issue for quite some time although not addressed until the introduction of the vortex coronagraph. This issue is especially problematic in the NIRC L-band due to the large band pass of the L' filter. It has been discovered that the primary cause of the dispersion is the IR dichroic as it had been installed improperly, with a significant $\sim 20 \%$ elongation on the L' PSF. To avoid this chromatic elongation, the dichroic plate had to be rotated $180^{\circ}$ but this caused again a pupil misalignment, so the same coordinated OAPs re-adjustment as with the case of the K-mirror was needed. Eventually, both the rotation of the dichroic and OAPs re-alignment caused an additional $\sim 250 \mathrm{~nm}$ astigmatism in the science path which is added to the Non Common Path Aberration (NCPA) calibration.

A remaining concern is that upon re-alignment of the $\mathrm{AO}$ bench components the NIRC2 field distortion and astrometric model has changed and should be re-done on-sky (e.g. as described in Ref. 14) since no grid hole is now available within NIRC2 .

\subsection{The AGPM integration}

For systems already equipped with a Lyot-style coronagraph, the upgrade to a vortex design simply requires replacing the focal-plane coronagraphic element with the AGPM, and a possible alteration to the Lyot stop to better match the vortex design.

The project to install a Vortex mask in NIRC2 was led by E. Serabyn (JPL) and it is a collaboration between JPL, CIT (led by D. Mawet), the European VORTEX group (led by O. Absil from U. Liège) and W.M. Keck Observatory (WMKO, led by P. Wizinowich). Part of this team has also been involved in the installation of Vortex masks at Palomar, ${ }^{8}$ VLT $^{4}$ and LBT. ${ }^{5}$ The vortex FPM was installed in NIRC2 in March 2015 replacing, at the focal plane, the grids of holes used for focusing and distortion tests on the focal Slit Mask Stage (SLS), which also holds the spectroscopic slits and a number of conventional (pure amplitude) coronagraphic masks which remain within NIRC2 and are still available for use.

Keith Matthews (CIT) led the service mission to install the vortex FPM on the slot previously occupied by the SLS. This upgrade took three weeks, but once the NIRC2 cryostat was warm it took only one day to open it and install the new vortex FPM. This operation was performed in the Keck II AO enclosure, which it is not a graded clean room but is continuously maintained dust free. For this occasion extra-measures were adopted: $\mathrm{AO}$ room underwent an additional exhaustive cleaning, filters in AO room were switched on and all personnel wearing appropriate clothing (tyvex suits, shoe coverings, hair caps and face masks).

Since this was a demonstrator project, in order to be cost-effective and minimize risks there was not attempt to consider an optimized Lyot stop and, instead, use the incircle pupil mask already in NIRC2 (bottom left panel in Fig. 2). As we later discovered when testing the system on-sky, we still suffer from leaks around from the 

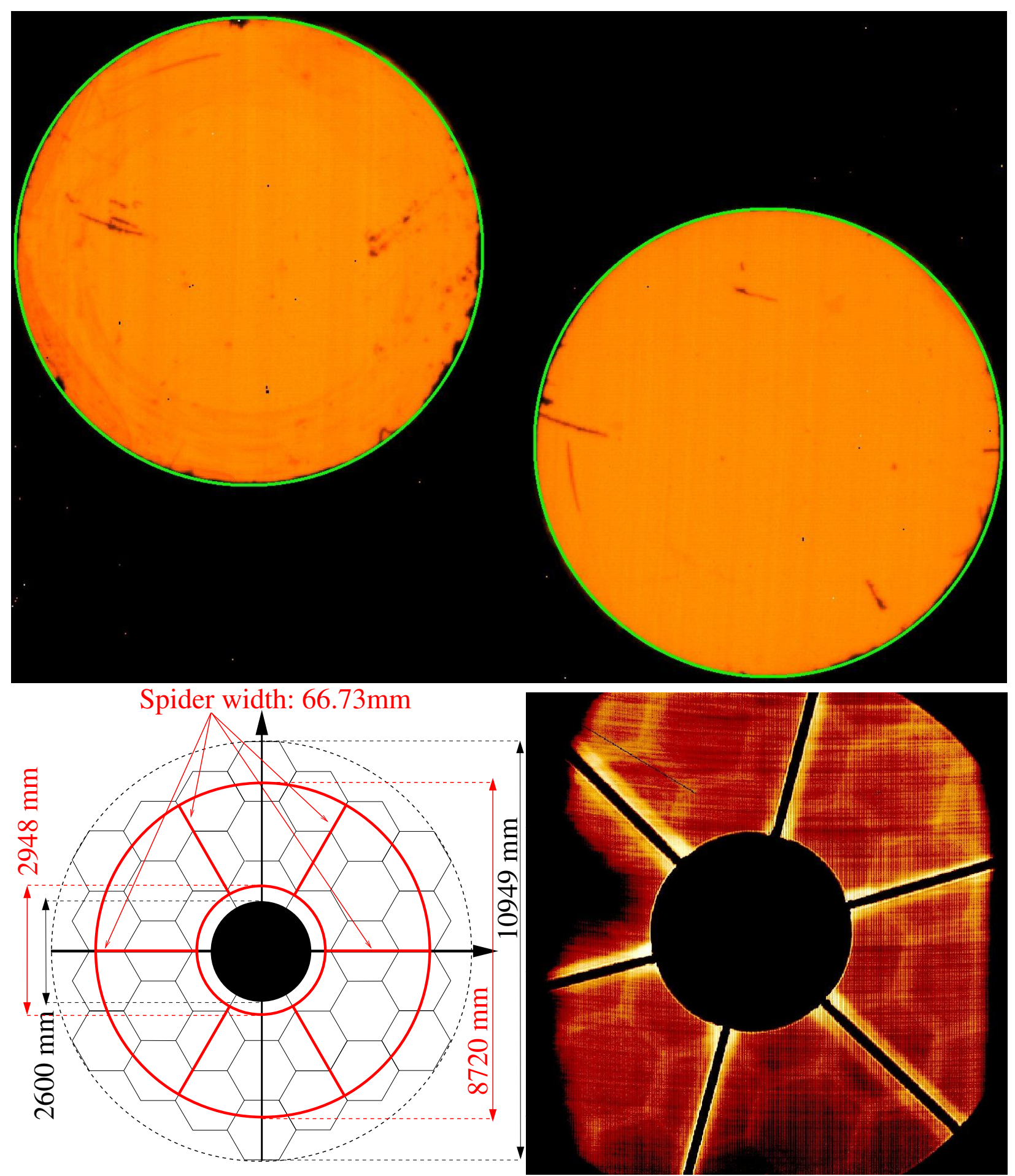

Figure 2. Top: The FPM with the two vortices on display. The green contour identifies visually the boundaries of each of the vortices which on sky subtend 12.1 arcsec and they lie $\sim 14.35$ arcsec from each other. Bottom Left: Diagram showing the relative dimensions of the Lyot mask when projected onto the telescope primary mirror (which defines the telescope pupil). Bottom Right: Image of the Lyot mask well aligned with respect to the primary mirror; please notice the residual leakage around the spiders. 
spiders, specially on the joints with the secondary mirror structure. An optimized Lyot mask would attenuate this and improve the achievable contrast ratio.

The top image in Fig. 2 shows the vortex patterns on the FPM (top image), the green circles are visual fits to determine the angular extents of both masks: 12.1 arcsec. The original purpose for the two vortex patterns on the same FPM was to optimize efficiency by nodding between one vortex pattern to the other so no time would be spent on sky background acquisition. However, this has not yet been attempted as it is deemed not feasible for two main reasons. First the accuracy of repositioning the spot after offseting from one mask to the other is not guaranteed given the accuracy of the Field Steering Mirrors (FSM) used in the acquisition system of the AO system WFS. Second, it is only possible to have both vortex patterns if resorting to the coarse plate scale of $40 \mathrm{mas} /$ pixel in which case we are at the Nyquist sampling of the L' PSF. While the former constrain may be coped with a more aggressive implementation of the pointing control algorithm (see Section 2.4), our commissioning tests and science programs executed so far demanded relying on the finest pixel scale. From the cosmetics of each vortex pattern on Fig. 2 our initial tests considered the mask on the bottom (a.k.a. vortex1) although posterior tests indicated slightly better performance with vortex2 (the pattern on the top).

In Fig. 3 we show an on-sky image in L' with NIRC2 and the vortex mask inserted, the target star was not centered on the mask. An unexpected effect is that the center of the mask glows at thermal IR bands, while the textbook effect of a vortex coronagraph is to create a dark hole in a flat field. This turns to be really convenient as it is a great aid to position the star to be attenuated pretty close to the center of the mask and then the fine tuning positioning can be performed by QACITS (see Section 2.4). The central glow of the vortex phase mask is most probably due to the fact that the vortex is located upstream the cold stop in NIRC2. It is understood to come from thermal emission of warm structures located outside the geometric pupil of the telescope,which gets partially diffracted back inside the pupil when passing through the vortex center. A more detailed description of this effect is presented in Ref. 15.

\subsection{Pointing control with the vortex at NIRC2: deployment of QACITS}

If one of the strongest benefits of a vortex coronagraph is its small IWA, it is also very sensitive to tip/tilt errors, requiring precise pointing control. Previous works ${ }^{4}$ suggest that in order to maintain its high contrast capability, the centering of the star on the AGPM must be within ${ }^{\S}(\lambda / D) / 10 \simeq 8.9$ mas. To achieve this level of centering on the vortex mask we deployed the "Quadrant Analysis of Coronagraphic Images for Tip-tilt Sensing" (QACITS) proposed in Ref. 16. Very briefly, QACITS provides an an estimate of the tip/tilt error by comparing the true image of the attenuated starlight with an optical misalignment model. This tip-tilt estimate is continuously recorded from the most recently acquired image and a dedicated low bandwidth tip-tilt loop recenters the image.

From the perspective of the implementation of QACITS, the problem we faced at NIRC2 was the lack of enough accuracy on the pointing on the Keck AO system based on the acquisition mechanisms in the WFS. At Keck II AO in Natural Guide Star (NGS) mode targets are acquired on the WFS by means of a pair of coordinated Field Steering Mirrors (FSMs) with an accuracy of $\sim 20$ mas, which results insufficient to meet the $\sim 8$ mas $r m s$ pointing accuracy requirement. Notice also this is one of the reason why the initial purpose of engraving two vortex patterns on the FPM to increase observational efficiency (see Section 2.3) has not been achieved: on performing small dithers or offsets usually well managed by the AO system in NGS mode we can not rely on bringing the star back to the vortex mask center with the required $\sim 8$ mas. We still need to further investigate this dithering/offset issue in detail for a possible future implementation. The solution to this problem was to correct for the misalignments by resetting the centroid offsets of the adaptive optics system. This allows for exquisite pointing control. While care must be taken not to exceed the linear regime, in which case we would suffer from a huge image quality loss.

The commissioning of QACITS on NIRC2 was successfully achieved on the second night we acquired data with the vortex on NIRC2 and it was the first light ever of QACITS on-sky. During our first commissioning run QACITS was able to stabilize the star on the mask center with accuracies $\sim 2$ mas, outperforming our initial goals

\footnotetext{
${ }^{\S} \mathrm{L}$ ' central wavelength is $\lambda=3.776 \mu \mathrm{m}$, and the diameter to be considered is the incircle pupil mask diameter $D=8.792 \mathrm{~m}$
} 


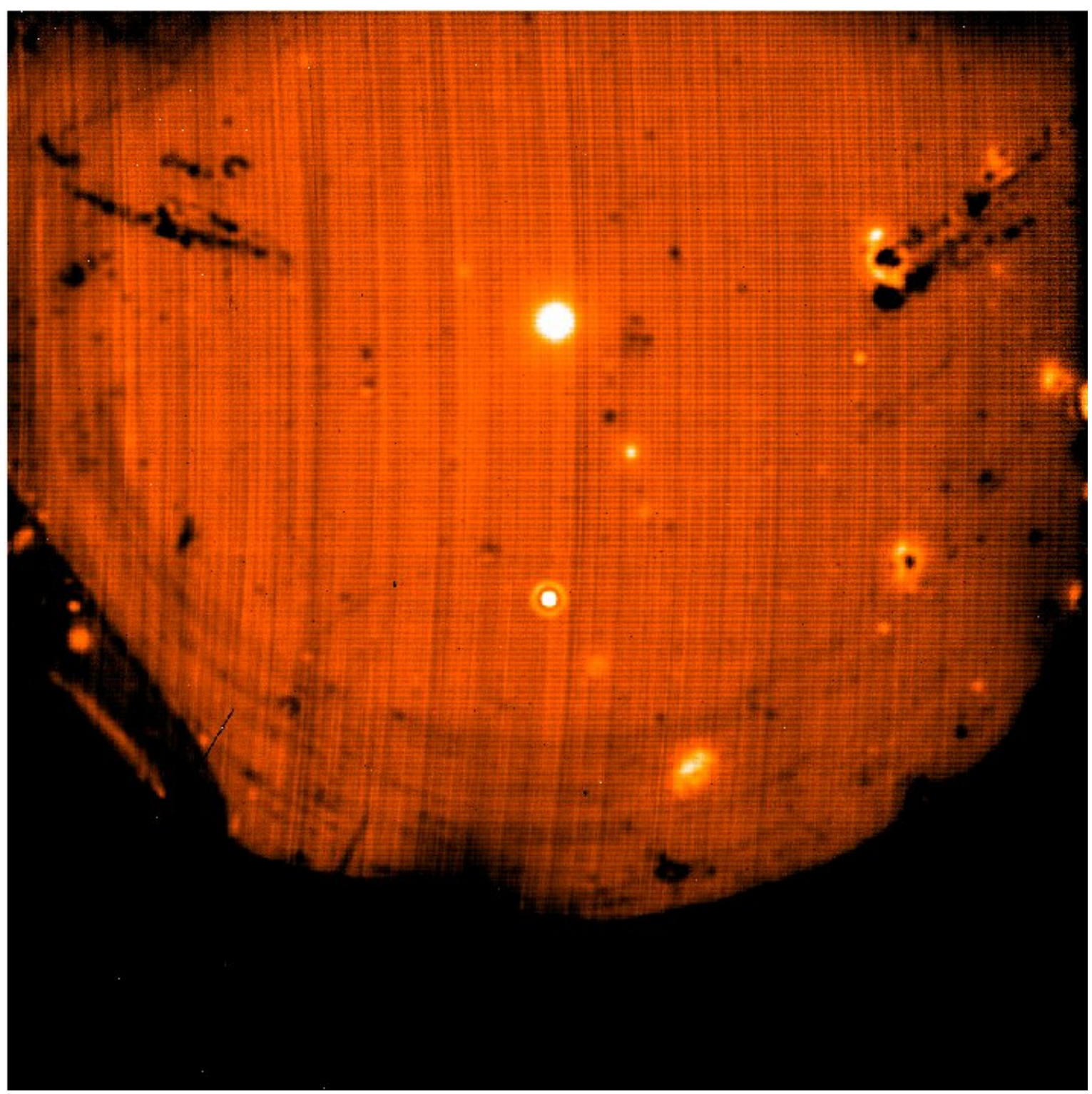

Figure 3. On sky image with vortex center glowing and target to the bottom of the mask. Notice the good PSF quality with the distinctive first Airy ring (in this image the AO loop has already been closed).

to provide stabilized images within $\sim 8$ mas, as required by the $(\lambda / D) / 10$ criterion. Continuous improvements during subsequent engineering runs from July 2015 until October 2015 have ended up with a very consolidated version which has also been offered ${ }^{18,19}$ to the Keck community in shared risk mode for the period 2016B (see details in Section 4). A dedicated account on the commissioning of QACITS on NIRC2 is given in this conference in Ref 17 .

\subsection{Fine wavefront control with speckle nulling at NIRC2}

As part of the optimization path in this project, we have considered speckle nulling as a means to further improve the high contrast capabilities of the vortex FPM at NIRC2. Part of the team had already accumulated significant experience with this technique at the Hale 200-inch telescope. The ultimate goal of speckle nulling is to attenuate the effects of static aberrations on the system which manifest as speckles on the post-coronagraphic images and can easily be mistaken by false positive detections. The interested reader is referred to Ref. 20 for a detailed 

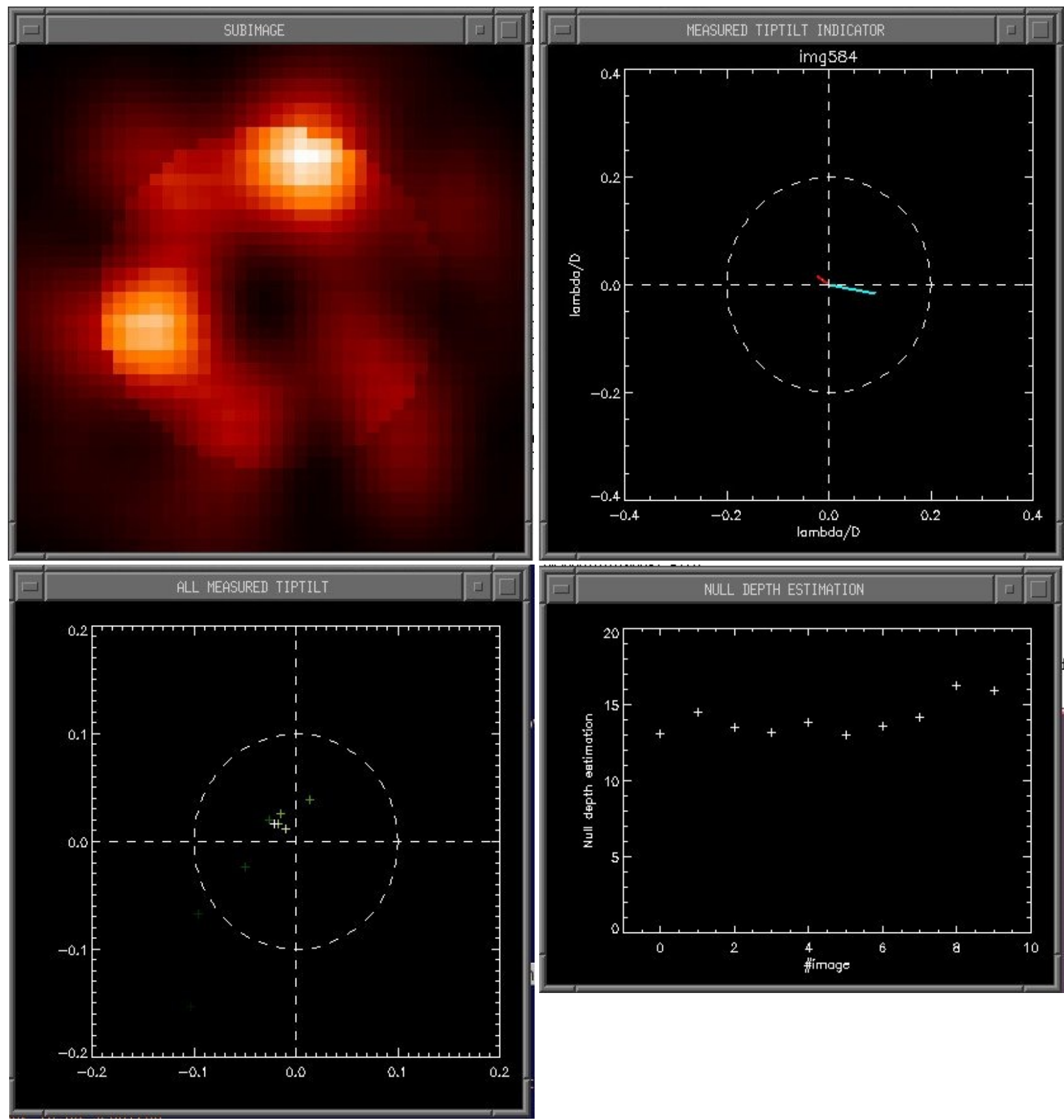

Figure 4. Displays opened during execution of QACITS. The top left displays zooms into the "SUBIMAGE" (sky subtracted) used by the QACITS estimator; the inner circle of radius 0.1 lambda/D is highlighted by $30 \%$. The top right window displays the current tip-tilt value using two different estimators: the blue vector uses the signal from the inner part of the image, the red vector uses the outer part of the image; the green dashed vector is the one that QACITS is currently using (in this case it can hardly bee seen but is superimposed on the red vector, i.e. using the outer signal). The bottom left display shows the history of tip-tilt values in the currently executed sequence of images. The bottom right panel provides an estimation of the null depth (ratio of the flux integrated over the central disk of the coronagraphic image and off-axis PSF). For further details see Refs. 16, 17.

account on the theory underlying this method, the pros and cons of speckle nulling as well as detailed preliminary results achieved using the internal calibration sources at both Palomar and Keck. Within the context of this 
paper we only sketch the steps required to have this feature in our system.
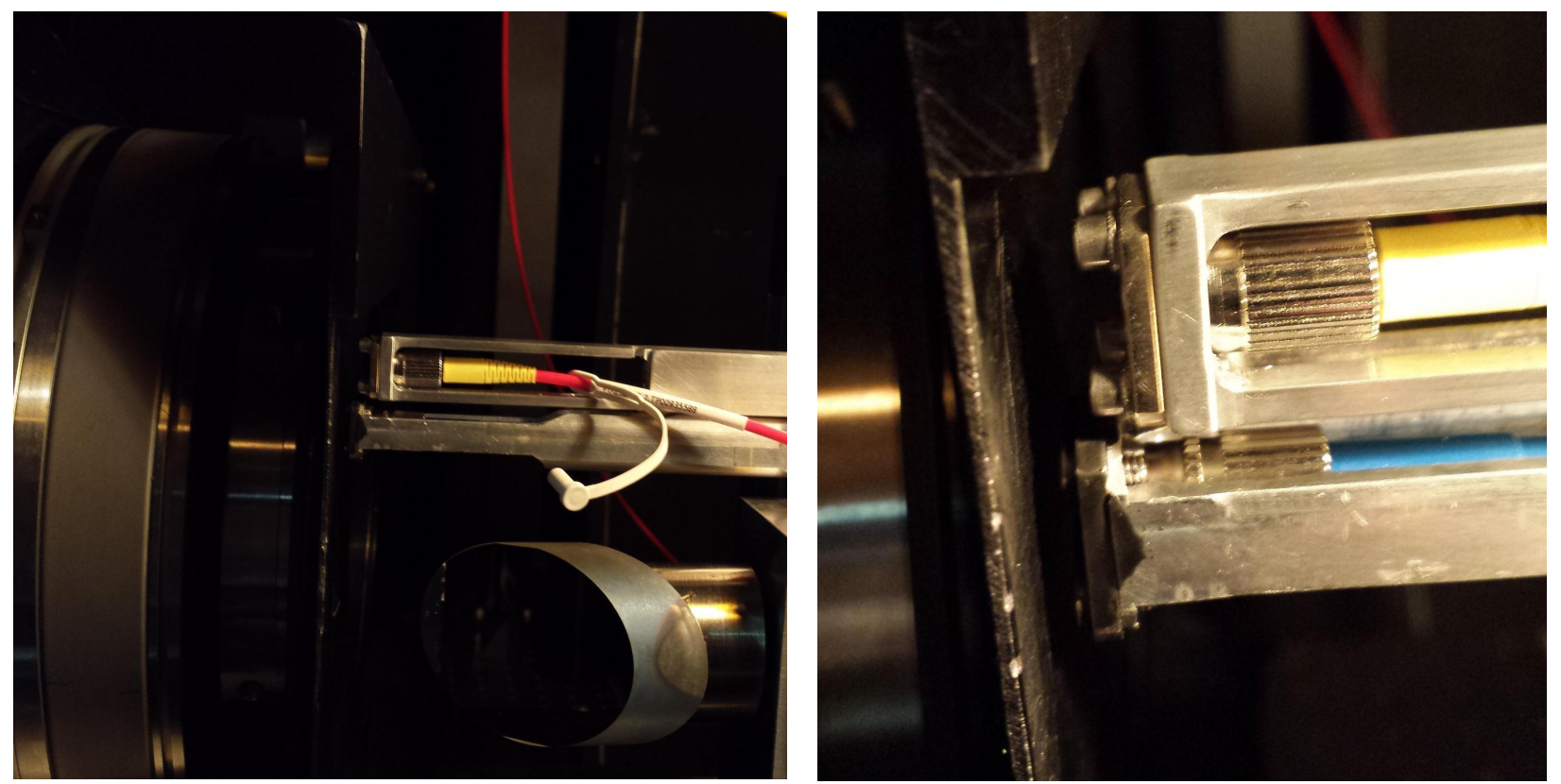

Figure 5. Left: Image of fiber holder with the standard calibration fiber on the bottom and the new chuck holding the IR source on top.Right: Zooming in the fiber head where it is visible both the lateral displacement from each other (about $22.1 \mathrm{arcsec}$ ) and the fact the fibers are not confocal. The consequence of this set up translates in large overheads during calibration of the system in L' band before handing over the system to the speckle nulling code.

The code used here is an adaptation to the specifics of Keck of a Python-based code by M. Bottom in Ref. 20. This speckle nulling implementation requires Python $2.7+$ which, at the moment of deployment at Keck, was not available on any of the operational servers controlling/communicating with either the AO or NIRC2 systems. Our first implementation of speckle nulling assumes no update of the speckle solution during observation; instead the speckle nulling solution as of this moment is integral part of the calibration of the AO/vortex system which needs to be loaded only once at the beginning of the observations. This means there is no bandwidth requirement to apply and/or update any solution and its practical consequence is that we could deploy the Python code on a virtual machine at WMKO HQs. In order to be able to communicate the virtual machine with both the Keck II AO system we needed to deploy the Python wrapper to the KTL API.

On the hardware side we experienced more issues. As mentioned in Section 2.1, for calibration purposes the Keck II AO system uses a white source to be observed on NIRC2 with a narrow Fe II band filter $(\lambda=$ $1.6455 \pm 0.0256 \mu \mathrm{m})$ and on the WFS on a broad band typically associated to the Bessel-Johnson R band. The core of the calibration scheme on a NGS consist on optimizing the image quality on the NIRC2 focal plane with an automated Modified Gerchberg-Saxton (MGS) algorithm. This Non-Common Path Aberration (NCPA) solution is then transferred to the calibration of the WFS by redefining its centroid offsets. However, in order to add to this standard NCPA solution the slight modifications imposed by speckle nulling, we need access to a source that is visible both by the WFS (in the $\sim \mathrm{R}$ band) and on L' band. The IR fibers deployed at the beginning of the project were only single mode above $2.3 \mu \mathrm{m}$. The solution was to add an additional chuck on the holder of the calibration fiber on the AO bench in order to have simultaneously light at R and L' bands. Although the solution is working, it is not an optimal implementation since both sources are located at different locations on the NIRC2 FoV (22.1 arcsec apart) and, due to installation constraints, they are not confocal. The implications of this solution is the system needs to be first calibrated in the standard fashion and then a lengthy calibration process is required to bring the IR fiber in focus with a good image quality in $\mathrm{L}$ band $\left(\mathrm{SR}_{L} \geq 90 \%\right)$. A solution could be a photonic crystal fiber behaving as a single mode transmitter over the $\mathrm{R}$ to $\mathrm{L} / \mathrm{M}$ spectrum. 


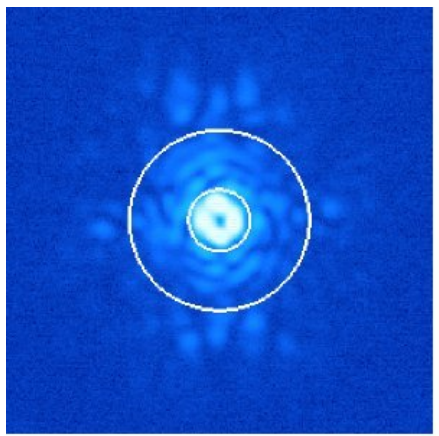

(a) Initial speckle field

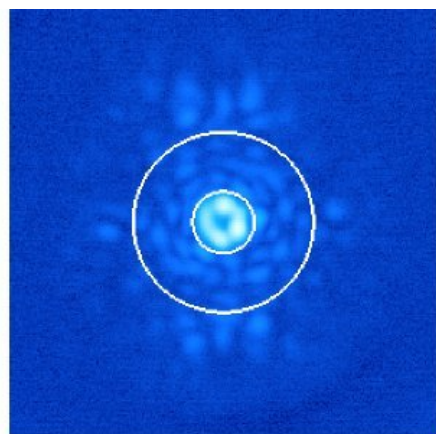

(b) Fourth iteration

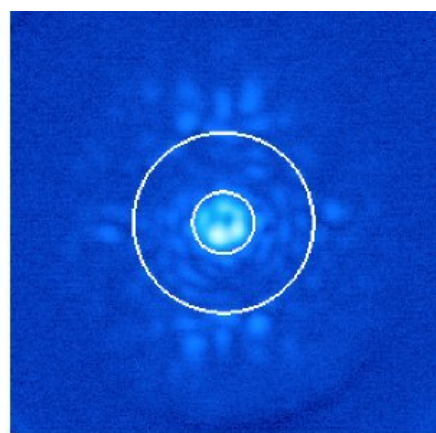

(c) Ninth iteration

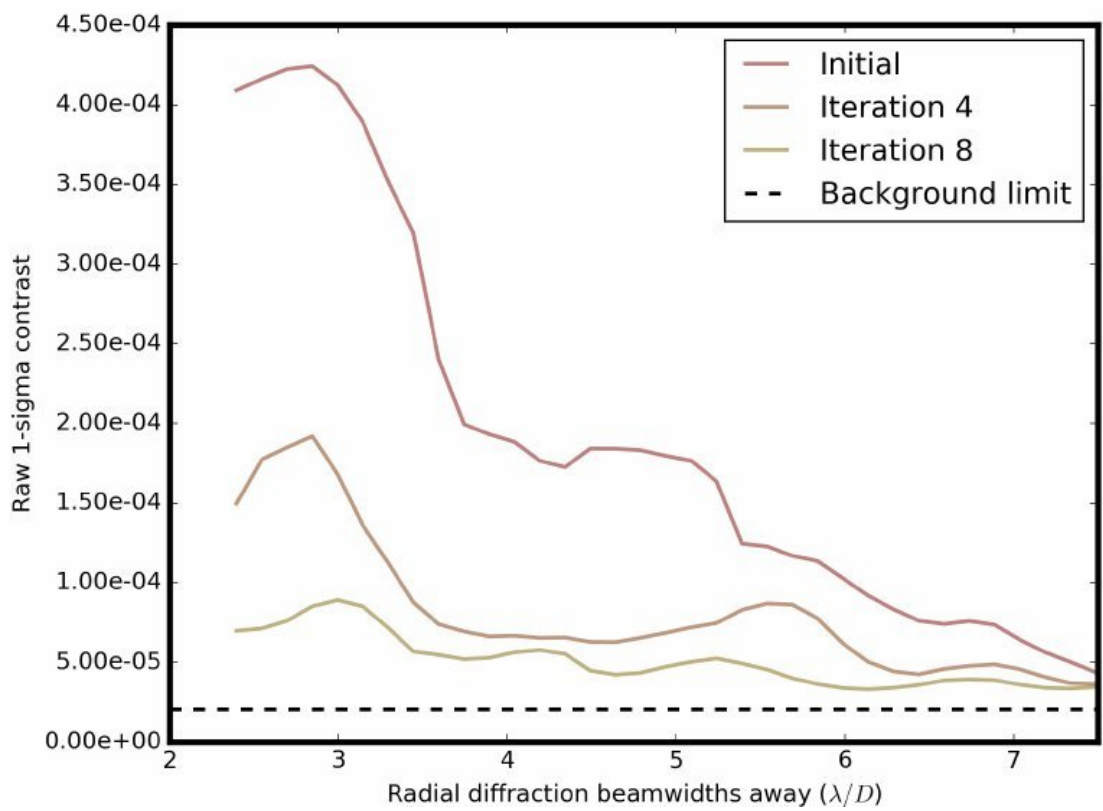

Figure 6. Taken from Ref. 20 to illustrate the contrast gain with the speckle nulling. Coronagraphic speckle nulling in L-band on NIRC2, using the internal white light source of the adaptive optics system. The value of $\lambda / D$ is $\sim 80 \mathrm{mas}$. (a) The initial results of PSF correction using image sharpening leaves many speckle aberrations in the focal plane. (b) Four iterations of speckle nulling remove. (c) Nine iterations of speckle nulling. The white lines demarcate the control region, with the outer line at approximately the limit of the control bandwidth of the deformable mirror. Bottom: The contrast improvement in this case is a factor of 5-10, and gets within a factor of 2-3 of the background limit. The contrast curve is defined in the usual way, with the standard deviation (i.e., $1 \sigma$ ) of surface brightness at each radial separation being used to generate the curve, and normalized by dividing by the peak flux of the non-coronagraphic PSF. The background limit is determined by the contrast in a region of the detector 100s of $\lambda / D$ away. The preprocessing steps performed on the data only consist of dark subtraction and flat-fielding.

\section{NIRC2 VORTEX CORONAGRAPH PERFORMANCE}

A detailed account of the data harvested using vortex coronagraphs in different 8-10 m class telescopes is provided in this conference in Ref. 15. Regarding the results achieved with the vortex at the NIRC2 on the Keck II telescope as of today, the most representative data has been acquired on October 24 th 2015 on the benchmark target ${ }^{12,13}$ HR8799. For this sequence the target was observed for nearly two hours around zenith (except for a few minutes around the zenith blind spot). HR8799 has an R-band magnitude $m_{R}=5.96$ that results in excellent AO correction. In the scientific L' band HR8799 has $m_{L}=5$ and the target was observed at elevations above $81^{\circ}$ (average air mass throughout the observation was only 1.014) with an excellent average seeing of $0.733 \pm 0.018$ arcsec and seeing dispersion 0.16 arcsec as measured towards zenith with a DIMM monitor every 90 seconds. 


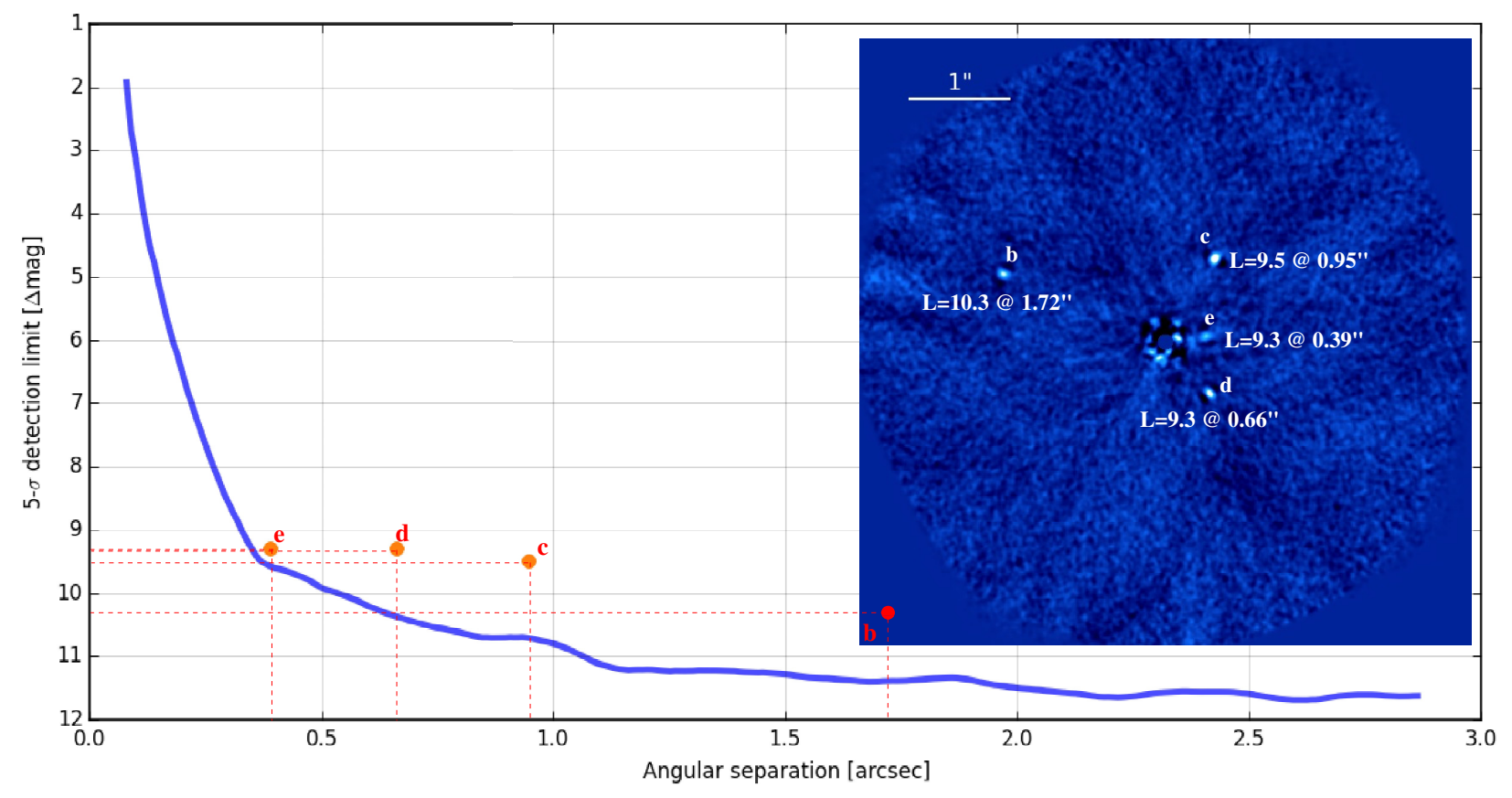

Figure 7. 5-sigma contrast curves on HR8799 on what as of today is the best performance achieved with the vortex at NIRC2. Inset on top right corner is the result of nearly two hours of total exposure time and it was processed with the VIP package using PCA-ADI.

A long 2-hr observation sequence on HR8799 was made using multiple 25 second exposures, on each of them running QACITS and Speckle Nulling (see Section 2.5). The observations were carried out in vertical angle mode (i.e.. the pupil was kept fixed by the AO K-mirror rotator) in order to improve the contrast via Angular Differential Image (ADI) . The contrast curves are provided in Figure 5. The final contrast curves were generated using the PCA-ADI algorithm in the python-based VIP post-processing package . It is worth mentioning a 10.2 magnitude contrast gain at $\sim 0.3$ arcsec.

More modest, yet still impressive, contrast values are achieved when observing dimmer targets. The contrast at 0.5 arcsec reaches a plato and remains constant with increasing angular distance since the background sets the contrast limit at these magnitude values. The first target corresponds to a bright NGS for which we expect very good high-order correction by the AO system as in the above case with HR8977. The second and third targets have similar birghtness $\left(m_{R}=12.3\right.$ and $\left.m_{R}=12.4\right)$ and we approach the limit on which the AO system perfroms equally if using NGS or LGS. The last target corresponds to a dim $m_{R}=13.29$ and under normal NIRC2 imaging programs it could be argued on the benefits of directly considering Laser Guide Star (LGS) $\mathrm{AO}$ correction. The data corresponding to targets in Table 1 was acquired under excellent seeing conditions for targets \#2 and \#4 but due to the declination of the targets at low elevations $\|$ which corresponds to effective seeing values of $0.52 \operatorname{arcsec}$ for and $0.65 \operatorname{arcsec}$ for $m_{R}=12.3$ and $m_{R}=13.29$ targets, respectively. Targets in Table 1 are part of an on-going $\mathrm{M}$ dwarf survey and all of them were observed under the same exposure time conditions: 30 frames, each frame with 60 coadds and each ocadd is 0.5 seconds (in order to reduce the risk of sautration due to high L' sky background) for a total open shutter time of 900 seconds.

IThe Vortex Imaging Processing (VIP) is the suggested data reduction package for vortex data. It is implemented in python and it is freely available at https://github.com/vortex-exoplanet

$\|$ Seeing values from DIMM refer at zenith distance and at $0.5 \mu m$ wavelength. Seeing scales with wavelength $(\lambda)$ and $\operatorname{airmass}(z)$ as $z^{3 / 5} \lambda^{1 / 5}$ 
Table 1. Representative contrast values with the vortex at NIRC2 from an on-going survey of M dwarfs

\begin{tabular}{|c|c|c|c|c|c|c|}
\hline Target ID & $m_{R}$ & $m_{L}$ & Elevation & DIMM seeing & Contrast @ 0.3 arcsec & Contrast @ 0.5 arcsec \\
\hline$\# 1$ & 9.3 & 7.3 & $77^{\circ}$ & N.A. & $6.0 \times 10^{-4}$ & $4.3 \times 10^{-4}$ \\
\hline$\# 2$ & 12.3 & 8.1 & $54^{\circ}$ & 0.48 arcsec & $7.3 \times 10^{-3}$ & $5.4 \times 10^{-3}$ \\
\hline$\# 3$ & 12.4 & 9.0 & $40^{\circ}$ & N.A. & $5.9 \times 10^{-2}$ & $6.9 \times 10^{-3}$ \\
\hline$\# 4$ & 13.3 & 10.0 & $61^{\circ}$ & 0.59 arcsec & $2.3 \times 10^{-2}$ & $8.0 \times 10^{-3}$ \\
\hline
\end{tabular}

\section{CONCLUSIONS AND FUTURE DEVELOPMENTS \& PLANS.}

Using the experience accumulated with vortex coronagraphs installed on the Hale, VLT and LBT telescopes, a demonstration project was initiated by the end of 2014 with the goal of installing a vortex vector coronagraph at the NIRC2 instrument fed by the Keck II AO system. This project aimed at taking advantage of the excellent image quality provided by the combination of the extraordinary site conditions at Maunakea and the well documented and established Keck II AO performance, the small inner working angle and high suppression of the vortex coronagraph, coupled with the more forgiving planet-to-star contrast ratios in the thermal infrared, more than compensate for the higher sky background at longer wavelengths. All these factors indicated that a vortex at Keck would provide a very competitive high-contrast imaging capability.

The vortex project at Keck has been a fast track project: initial conversations between PI and co-PIs started on February 2014, WMKO directorate appointed a Project Manager for this project as of end of April 2014, hardware installation took place on March 2015 and first light with the vortex and with QACITS were successfully achieved on June 8-10 2015. This quick development was due thanks to one of the many benefits of the vortex coronagraph (i.e. ease of installation in systems already deployed with a classic Lyot coronagraph) and to the experience accumulated by a significant part of the team in deploying vortex FPMs at other telescopes.

As of today the results achieved with the vortex at NIRC2 have fulfilled our expectations in terms of high contrast achieved with the largest optical/IR telescope equipped with AO. This success motivated in February 2016 the Keck Science Steering Committee (KSSC) to promote the new vector vortex coronagraph from a demonstration facility to shared-risk NIRC2 operation during period 2016B without speckle nulling. In summary, the vortex at NIRC2 has allowed to implement a fast track and very cost effective high-contrast imaging capability at Keck able to compete with current state-of-the art planet imaging systems like GPI and SPHERE at NIR wavelengths.

Current programs are underway with the goals of further improve the system in terms of:

Vortex characterization in $\mathbf{M}$ band. So far all our efforts have been concentrated in L' band but the vortex FPM should be providing good high contrast capabilities in M band as well.

Observational efficiency. Despite the current limitations on how to perform offseting on both vortex patterns on the same FPM we still believe it is worthwhile investigating an increase of the system efficiency by using both vortices. We need to investigate whether a more sophisticated interface of QACITS with the way the AO system may handle dithers/offsets in an efficient way to solve for the limitations imposed by the accuracy on the repositioning with the pointing system in the WFS in the Keck II AO when considering NGS operation. If this is possible it needs to be justified also the performance impact caused by the need to consider coarse pixel scales forcing to operate a high-contrast facility nearly at the Nyquist sampling limit.

Speckle Nulling full deployment and optimization. As of today, speckle nulling has been used several times at the cost of increased overheads during daytime calibration of the system. We expect to be able to significantly reduce these overheads the moment a suitable calibration source can be used to illuminate both the WFS (operating in visible wavelengths) and the NIRC2 instrument in L' and M bands. A tentative solution relying on photonic crystal fibers has been identified. 
Speckle Nulling on-sky optimization. The goal is to be able to run the speckle nulling calibration on-sky on the same star to be attenuated by the vortex. Despite the fact that the Keck II AO system sits on a Nasmyth platform ensures a high degree of stability of the AO system, an on-sky speckle nulling update would allow to handle efficiently with long-term speckles caused by changes on the optical configuration due by thermal and/or flexure effects which can not be anticipated during daytime calibration.

Optimization of the Lyot stop. Given the success of the current vortex system at NIRC2 and the fact we are still being penalized by the use on a non-optimal Lyot mask (i.e. the incircle pupil mask at NIRC2) we need to run a risk/benefit analysis on upgrading the Lyot mask.

\section{ACKNOWLEDGMENTS}

The W. M. Keck Observatory is operated as a scientific partnership among the California Institute of Technology, the University of California, and the National Aeronautics and Space Administration. The Observatory was made possible by the generous financial support of the W. M. Keck Foundation.

Part of this work was carried out at the Jet Propulsion Laboratory, California Institute of Technology, under contract with NASA.

The research leading to these results has received funding from the European Research Council under the European Union's Seventh Framework Programme (ERC Grant Agreement n. 337569), and from the French Community of Belgium through an ARC grant for Concerted Research Action.

The authors wish to recognize and acknowledge the very significant cultural role and reverence that the summit of Maunakea has always had within the indigenous Hawaiian community. We are most fortunate to have the opportunity to conduct observations from this mountain.

\section{REFERENCES}

[1] Mawet, D., Riaud, P., Absil, O., and Surdej, J., "Annular Groove Phase Mask Coronagraph," ApJ 633, 1191-1200 (Nov. 2005).

[2] Mawet, D., Pueyo, L., Moody, D., Krist, J., and Serabyn, E., "The Vector Vortex Coronagraph: sensitivity to central obscuration, low-order aberrations, chromaticism, and polarization," in [Modern Technologies in Space- and Ground-based Telescopes and Instrumentation], Proc. SPIE 7739, 773914 (July 2010).

[3] Delacroix, C., Absil, O., Forsberg, P., Mawet, D., Christiaens, V., Karlsson, M., Boccaletti, A., Baudoz, P., Kuittinen, M., Vartiainen, I., Surdej, J., and Habraken, S., "Laboratory demonstration of a mid-infrared AGPM vector vortex coronagraph," A\&A 553, A98 (May 2013).

[4] Mawet, D., Absil, O., Delacroix, C., Girard, J. H., Milli, J., O’Neal, J., Baudoz, P., Boccaletti, A., Bourget, P., Christiaens, V., Forsberg, P., Gonte, F., Habraken, S., Hanot, C., Karlsson, M., Kasper, M., Lizon, J.-L., Muzic, K., Olivier, R., Peña, E., Slusarenko, N., Tacconi-Garman, L. E., and Surdej, J., "L'-band AGPM vector vortex coronagraph's first light on VLT/NACO. Discovery of a late-type companion at two beamwidths from an F0V star," A $\& A$ 552, L13 (Apr. 2013).

[5] Defrère, D., Absil, O., Hinz, P., Kuhn, J., Mawet, D., Mennesson, B., Skemer, A., Wallace, K., Bailey, V., Downey, E., Delacroix, C., Durney, O., Forsberg, P., Gomez, C., Habraken, S., Hoffmann, W. F., Karlsson, M., Kenworthy, M., Leisenring, J., Montoya, M., Pueyo, L., Skrutskie, M., and Surdej, J., "L'-band AGPM vector vortex coronagraph's first light on LBTI/LMIRCam," in [Adaptive Optics Systems IV], Proc. SPIE 9148, 91483X (July 2014).

[6] Jovanovic, N., Martinache, F., Guyon, O., Clergeon, C., Singh, G., Kudo, T., Garrel, V., Newman, K., Doughty, D., Lozi, J., Males, J., Minowa, Y., Hayano, Y., Takato, N., Morino, J., Kuhn, J., Serabyn, E., Norris, B., Tuthill, P., Schworer, G., Stewart, P., Close, L., Huby, E., Perrin, G., Lacour, S., Gauchet, L., Vievard, S., Murakami, N., Oshiyama, F., Baba, N., Matsuo, T., Nishikawa, J., Tamura, M., Lai, O., Marchis, F., Duchene, G., Kotani, T., and Woillez, J., "The Subaru Coronagraphic Extreme Adaptive Optics System: Enabling High-Contrast Imaging on Solar-System Scales," PASP 127, 890-910 (Sept. 2015).

[7] Swartzlander, Jr., G. A., Ford, E. L., Abdul-Malik, R. S., Close, L. M., Peters, M. A., Palacios, D. M., and Wilson, D. W., "Astronomical demonstration of an optical vortex coronagraph," Optics Express 16, 10200 (June 2008). 
[8] Mawet, D., Serabyn, E., Liewer, K., Burruss, R., Hickey, J., and Shemo, D., "The Vector Vortex Coronagraph: Laboratory Results and First Light at Palomar Observatory," ApJ 709, 53-57 (Jan. 2010).

[9] Dekany, R., Roberts, J., Burruss, R., Bouchez, A., Truong, T., Baranec, C., Guiwits, S., Hale, D., Angione, J., Trinh, T., Zolkower, J., Shelton, J. C., Palmer, D., Henning, J., Croner, E., Troy, M., McKenna, D., Tesch, J., Hildebrandt, S., and Milburn, J., "PALM-3000: Exoplanet Adaptive Optics for the $5 \mathrm{~m}$ Hale Telescope," ApJ 776, 130 (Oct. 2013).

[10] Macintosh, B., Graham, J. R., Ingraham, P., Konopacky, Q., Marois, C., Perrin, M., Poyneer, L., Bauman, B., Barman, T., Burrows, A. S., Cardwell, A., Chilcote, J., De Rosa, R. J., Dillon, D., Doyon, R., Dunn, J., Erikson, D., Fitzgerald, M. P., Gavel, D., Goodsell, S., Hartung, M., Hibon, P., Kalas, P., Larkin, J., Maire, J., Marchis, F., Marley, M. S., McBride, J., Millar-Blanchaer, M., Morzinski, K., Norton, A., Oppenheimer, B. R., Palmer, D., Patience, J., Pueyo, L., Rantakyro, F., Sadakuni, N., Saddlemyer, L., Savransky, D., Serio, A., Soummer, R., Sivaramakrishnan, A., Song, I., Thomas, S., Wallace, J. K., Wiktorowicz, S., and Wolff, S., "First light of the Gemini Planet Imager," Proceedings of the National Academy of Science 111, 12661-12666 (Sept. 2014).

[11] Beuzit, J.-L., Feldt, M., Dohlen, K., Mouillet, D., Puget, P., Wildi, F., Abe, L., Antichi, J., Baruffolo, A., Baudoz, P., Boccaletti, A., Carbillet, M., Charton, J., Claudi, R., Downing, M., Fabron, C., Feautrier, P., Fedrigo, E., Fusco, T., Gach, J.-L., Gratton, R., Henning, T., Hubin, N., Joos, F., Kasper, M., Langlois, M., Lenzen, R., Moutou, C., Pavlov, A., Petit, C., Pragt, J., Rabou, P., Rigal, F., Roelfsema, R., Rousset, G., Saisse, M., Schmid, H.-M., Stadler, E., Thalmann, C., Turatto, M., Udry, S., Vakili, F., and Waters, R., "SPHERE: a 'Planet Finder' instrument for the VLT," in [Ground-based and Airborne Instrumentation for Astronomy II], Proc. SPIE 7014, 701418 (July 2008).

[12] Marois, C., Macintosh, B., Barman, T., Zuckerman, B., Song, I., Patience, J., Lafrenière, D., and Doyon, R., "Direct Imaging of Multiple Planets Orbiting the Star HR 8799," Science 322, 1348 (Nov. 2008).

[13] Marois, C., Zuckerman, B., Konopacky, Q. M., Macintosh, B., and Barman, T., "Images of a fourth planet orbiting HR 8799," Nature 468, 1080-1083 (Dec. 2010).

[14] Yelda, S., Lu, J. R., Ghez, A. M., Clarkson, W., Anderson, J., Do, T., and Matthews, K., "Improving Galactic Center Astrometry by Reducing the Effects of Geometric Distortion," ApJ 725, 331-352 (Dec. 2010).

[15] Absil, O., Mawet, D., Karlsson, M., Defrère, D., Delacroix, C., Femenia Castella, B., Girard, J., Hinz, P., Huby, E., Matthews, K., Pantin, E., Seraby, G., Tristram, K., Wizinowich, P., Forsberg, P., Carlomagno, B., Christiaens, V., Forsberg, P. Gomez Gonzalez, C., Habraken, S., Jolivet, A., Milli, J., Piron, P., Reggiani, M., Surdej, J., Vargas Catalan, E., and O., W., "Three years of harvest with the vector vortex coronagraph in the thermal infrared," in [Ground-based and Airborne Instrumentation for Astronomy VI], Proc. SPIE 9908 (2016).

[16] Huby, E., Baudoz, P., Mawet, D., and Absil, O., "Post-coronagraphic tip-tilt sensing for vortex phase masks: The QACITS technique," A\&\&A 584, A74 (Dec. 2015).

[17] Huby, E., Absil, O., Mawet, D., Femenia-Castella, B., Bottom, M., Baudoz, P., Ngo, H., and Serabyn, E., "The QACITS pointing sensor: from theory to on-sky operation on Keck/NIRC2," in [Adaptive Optics Systems V], Proc. SPIE 9909, this conference proceedings (2016).

[18] Femenía Castellá, B., Huby, E., and Bottom, M., "Vortex at NIRC2: User Manual," Tech. Rep. Keck Adaptive Optics Note \#1104, W. M. Keck Observatory (2016).

[19] Huby, E., "How to run QACITS for the Vortex coronagraph at NIRC2 on Keck II," Tech. Rep. Keck Adaptive Optics Note \#1105, W. M. Keck Observatory (2016).

[20] Bottom, M., Femenia-Castella, B., Huby, E., Mawet, D., and Serabyn, E., "Speckle nulling wavefront control for Palomar and Keck," in [Adaptive Optics Systems V], Proc. SPIE 9909, this conference proceedings (2016). 